\title{
Receiver Operating Characteristics of GNSS Coarse/Acquisition Signals With Short Codes
}

\author{
Christoph Enneking, Felix Antreich, and André L. F. de Almeida
}

\begin{abstract}
Reliable signal acquisition with low computational complexity is an important design objective for the evolution of global navigation satellite systems (GNSS). Most GNSS signals consist of long pseudorandom noise (PRN) codes whose acquisition is expensive in terms of memory, computation time, and energy. As these resources are particularly scarce in the emerging mass-market user segment, dedicated coarse/acquisition (C/A) signals with short codes are being designed to keep the number of acquisition search bins low. However, reducing the code length degrades the receiver operating characteristic (ROC), as multiple access interference (MAI) from other C/A signals increases the probability of false alarm. MAI complicates the C/A signal design process considerably, because (quite different from stationary noise) it does not affect each bin of the search space in the same way. Taking into account the cyclostationarity of C/A signals, we propose a new randomized version of the spectral separation coefficient (SSC) as a simple yet accurate interference measure, which can be used for ROC performance evaluation and optimization. Accounting for MAI and other random degradation effects (e.g. data symbol transitions or finite search resolution), we establish a new methodology to assess the ROC for shorter and shorter PRN codes. Ultimately, our approach enables the C/A signal designer to minimize the PRN code length while ensuring a given target $\mathrm{ROC}$ performance.
\end{abstract}

Index Terms-Multi-user interference, global positioning system, time-to-first-fix.

\section{INTRODUCTION}

$\mathbf{S}$ IGNAL acquisition is a resource-hungry process for receivers of global navigation satellite systems (GNSS). A GNSS satellite transmits pseudorandom noise (PRN) code and navigation data which arrive at the receiver with low signalto-noise ratio, unknown code-phase, and unknown Doppler frequency. To acquire such a signal, the receiver must correctly detect that the signal is actually present at the receive antenna, and estimate the two unknown synchronization parameters code-phase/Doppler with coarse resolution [1]. The necessary 2-D search for correlation over a set of code-phase/Doppler hypotheses (bins) requires considerable computation time (if bins are searched sequentially), memory (if bins are searched in parallel), and in any case energy [2]-[4]. Besides these complexity measures, the global probability of false alarm (GPF) and the global probability of detection (GPD) indicate the reliability of the acquisition in case of the absence or presence of the signal, respectively [5], [6]. Together, GPF and GPD form the receiver operating characteristic (ROC). In

C. Enneking is with the German Aerospace Center (DLR), Oberpfaffenhofen, Germany (e-mail: christoph.enneking@dlr.de). A. L. F. de Almeida and C. Enneking are with the Federal University of Ceará (UFC), Fortaleza, Brazil (e-mail: andre@gtel.ufc.br). F. Antreich is with the Aeronautics Institute of Technology (ITA), São José dos Campos, Brazil (e-mail: antreich@ieee.org). case of a false alarm or missed detection, even more receiver resources are consumed (new acquisition, false initialization of tracking loops).

For the above reasons, acquisition is one of the computationally most expensive stages of a GNSS receiver. This poses a major challenge especially to mass-market consumer electronics such as mobile phones, asset trackers, or internet of things devices [3], [7]. Typically, such receivers are only occasionally prompted to provide positioning or navigation, so that they spend much of their duty cycle in acquisition mode. Moreover, they are built on very small integrated circuits with limited memory, but are expected to acquire several signals within few seconds and to economize the precious battery energy. A compromise between memory and computation time can be achieved, using serial, parallel, or hybrid search techniques [8], [9]. The consumed energy, however, is essentially a constant proportional to the number of bins and cannot be traded off at the cost of some other resource. Therefore, it is desirable to actually reduce the overall complexity of the search, i.e., the number of bins. While the number of Doppler bins is usually on the order of 1-30 due to the wide use of Assisted-GNSS by mass-market receivers, the number of code-phase bins is essentially proportional to the PRN code length [3].

We focus on an apparently simple transmitter side solution in this work: keeping the signal's PRN code short, with a length of 1023 chips or even less. Moreover, the signals should also have a low symbol rate to avoid a sensitivity loss due to symbol transitions [10]. For instance, the symbols could carry only absolutely necessary navigation data such as the GNSS time reference. Pursuing this approach, dedicated coarse/acquisition (C/A) signals are being designed [11]-[14] or re-designed [15], the successful GPS L1 C/A serving as a blueprint (and giving GPS a competitive edge in consumer GNSS [16]). As the authors of [15] point out, this combination of short PRN period and long symbol duration (more precisely, the repetition of more than one PRN code during the transmission of a single symbol) has an inconvenient implication. It leads to significant vulnerability to self-interference, i.e., multiple access interference (MAI) of C/A-on-C/A signals. It is known that this MAI can create a detectable false correlation peak in near-far scenarios [17]. Under nominal received power levels, by contrast, the signal designer should ensure, using appropriate models ore simulations, that the ROC is not degraded excessively by MAI.

The theory of spectral separation coefficients (SSCs) is a powerful tool to model the MAI between any two signals, e.g., between a local replica of the PRN code of interest and an interfering signal with another PRN code. While the standard 
SSC as originally proposed by Betz [18], [19] is only accurate for stationary MAI, more accurate versions (referred to as SSC-D hereafter) have been proposed which also work well with C/A signals [20]-[23]. The SSC-D takes into account fine features of the signals' power spectral density (PSD), and therefore depends on the instantaneous relative Doppler frequency between local replica and interferer. However, the statistical effect of MAI on the ROC in nominal conditions has not yet been investigated systematically. A straightforward application of the SSC-D simply requires considerable processing, as the MAI from a set of interfering satellites on each local replica of the 2-D search space would have to be evaluated. Doing this during a system optimization process, in which the PRN code length (and possibly other parameters) are under design, would be computationally prohibitive.

In this work, we propose a methodology to assess the impact of cyclostationary MAI on the ROC for any given PRN code length. Our approach is based on the SSC-D, but a randomized version thereof (SSC-R). A key assumption is that MAI can be approximated as independent and identicall distributed (i.i.d.) random variables over all bins. This approximation obviates the need to compute the SSC-D explicitly for each bin, but allows to work with one representative probability density function (PDF) instead. Our approach is a tradeoff between the accuracy of the SSC-D [20]-[22] and the simplicity of the SSC [18], [19], simple enough to use for optimization of system parameters. As an application example, we demonstrate how the proposed methodology can be used to minimize the PRN code length, while ensuring constraints on reliability and sensitivity. For simplicity, we only consider self-MAI in this work (i.e., all users have the same symbol rate and code length), but the approach can be easily generalized to intersystem-MAI using [22].

The rest of this paper is organized as follows. In Section II, we define the acquisition system, consisting of the received signal model, the generation of decision statistics, and the search strategy. In Section III, we discuss the probability of false alarm under various versions of the SSC, including the novel SSC-R. In Section IV, we model the probability of detection, taking into account MAI, symbol transitions, and finite search resolution. In Section V, the validity of the SSC$\mathrm{R}$ is demonstrated at the example of numerical simulations. Finally, we present an application of the proposed model for signal design in Section VI.

\section{System Model}

We consider $K$ satellite signals received over an additive white Gaussian noise (AWGN) channel, where one signal is to be acquired and the remaining $K-1$ signals are interference. For $P$ code bins and $Q$ Doppler bins, we describe the processing steps for the generation of $P Q$ decision statistics based on the same set of input baseband samples. An arbitrary but fixed number of coherent and noncoherent summations is considered. Subsequently, we briefly describe the search strategy for serial and parallel search.

\section{A. Baseband Signal}

The received signal in complex baseband notation is

$$
r(t)=\sum_{k=1}^{K} \sqrt{P_{k}} x_{k}(t)+w(t),
$$

where the $k$ th satellite contributes the signal $x_{k}(t)$ with power $P_{k}$, while the real and imaginary part of $w(t)$ are two independent white Gaussian noise processes each with twosided PSD $N_{0} / 2$. A model for $x_{k}(t)$ is shown at the top of Fig. 1. For $k=1, \ldots, K$, the satellite signals are given as

$$
x_{k}(t)=e^{\mathrm{j} 2 \pi \nu_{k} t+\mathrm{j} \varphi_{k}} \sum_{m=-\infty}^{\infty} s_{k}\left(t-m T_{0}-\tau_{k}\right) b_{k}[m] .
$$

The parameters $\tau_{k}, \nu_{k}$, and $\varphi_{k}$ denote to the unknown codephase, Doppler frequency, and carrier-phase, respectively. The signal $s_{k}(t)$ is a known code waveform, which repeats with code rate $1 / T_{0}$ and is modulated by a symbol sequence $b_{k}[m] \in\{-1,+1\}$. We consider only direct-sequence codedivision multiple access (DS-CDMA) waveforms of the structure

$$
s_{k}(t)=\sum_{j=0}^{N_{c}-1} c_{k}[j] h\left(t-j T_{c}\right),
$$

where $c_{k}[j]$ is a known PRN code, and $h(t)$ is a realvalued analog pulse shape (for instance, a rectangular pulse). Therefore, the code period is equal to $T_{0}=N_{c} T_{c}$. For $k=1, \ldots, K$, the code-phase, carrier-phase, and Doppler frequency are assumed to be independent random variables on the intervals $\left(-T_{0} / 2, T_{0} / 2\right),(-\pi, \pi)$, and $\left(-F_{0} / 2, F_{0} / 2\right)$, respectively, with some Doppler span $F_{0} \geqslant 0$.

The statistical properties of the PRN code $c_{k}[j]$ and the symbol sequence $b_{k}[\mathrm{~m}]$ are an important aspect of our analyses, as they can have a fundamental impact on the distribution of MAI. We model the PRN code $c_{k}[0], \ldots, c_{k}\left[N_{c}-1\right]$ as a "coin-flip" sequence of length $N_{c}$ (i.e., $N_{c}$ i.i.d. binary random variables which assume values $\{-1,+1\}$ with equal probability). The symbol sequence $b_{k}[m]$ is not, in general, a coinflip sequence of infinite length. Instead, we use the slightly more general model $b_{k}[m]=d_{k}\left[\left\lceil\left(m-\vartheta_{k}\right) / M\right\rceil\right]$, where $d_{k}[m]$ is an infinite coin-flip sequence, $\vartheta_{k} \in\{1, \ldots, M\}$ is a uniformly random initial symbol-phase, and $M \in \mathbb{N}$. Thus, a symbol transition may only occur every $M$ elements of $b_{k}[m]$. PRN code and symbol sequences are mutually independent, and also independent for $k=1, \ldots, K$. Note that we model both as truly random, although in fact PRN code will always and symbols (e.g. secondary code) may sometimes be pseudorandom.

It is important to distinguish between the symbol rate $1 /\left(M T_{0}\right)$ and the code rate $1 / T_{0}$. A few typical setups are worth mentioning: (i) balanced: $M=1$; (ii) quasi-pilot: $M \gg 1$; (iii) pure pilot: $M \rightarrow \infty$. The balanced configuration (i) is typical for modernized civil GNSS services such as the Galileo Open Service [24]. In this work, we will focus on (ii) and (iii), as they are the most attractive options for C/A signals, and the most prone to MAI. 

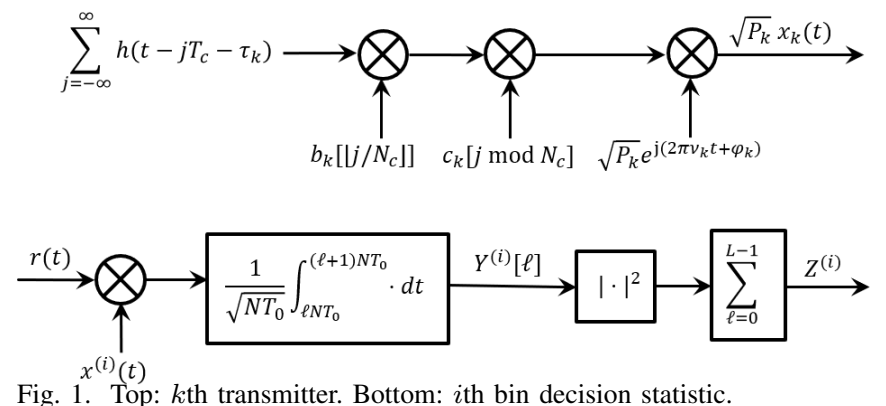

\section{B. Decision Statistics}

Without loss of generality, we consider $k=1$ as the signal of interest (SOI). The acquisition task is specified as follows. Decide for either of the following hypotheses:

- $H_{0}$ : the SOI is absent $\left(P_{1}=0\right)$;

- $H_{1}$ : the SOI is present $\left(P_{1}>0\right)$;

additionally, if the decision is taken for $H_{1}$, select a coarse estimate for $\tau_{1}$ from a set of code-phase candidates $\mathcal{X}_{\tau}$, and a coarse estimate for $\nu_{1}$ from a set of Doppler candidates $\mathcal{X}_{\nu}$.

These candidate sets form a 2-D grid of bins with $P$ codephases and $Q$ Doppler frequencies, distributed uniformly over the uncertainty intervals $\left(-T_{0} / 2, T_{0} / 2\right)$ and $\left(-F_{0} / 2, F_{0} / 2\right)$, respectively. Thus we have

$$
\begin{aligned}
& \mathcal{X}_{\tau}=\left\{-\frac{T_{0}+\Delta \tau}{2},-\frac{T_{0}+3 \Delta \tau}{2}, \ldots, \frac{T_{0}-\Delta \tau}{2}\right\}, \\
& \mathcal{X}_{\nu}=\left\{\frac{-F_{0}+\Delta \nu}{2}, \frac{-F_{0}+3 \Delta \nu}{2}, \ldots, \frac{F_{0}-\Delta \nu}{2}\right\},
\end{aligned}
$$

with code-phase spacing $\Delta \tau=T_{0} / P$ and frequency spacing $\Delta \nu=F_{0} / Q$. We use a linear index $i \in\{1, \ldots, P Q\}$ to refer to the 2-D $\operatorname{bin}\left(\tau^{(i)}, \nu^{(i)}\right) \in\left(\mathcal{X}_{\tau} \times \mathcal{X}_{\nu}\right)$. We define the correct bin with index $i=1$ as the bin that deviates the least from the true parameters in the sense that

$$
\left|\tau^{(1)}-\tau_{1}\right| \leqslant \Delta \tau / 2 \text { and }\left|\nu^{(1)}-\nu_{1}\right| \leqslant \Delta \nu / 2 .
$$

It is easily checked that this assignment is unique, i.e., there is always one correct bin with probability one. ${ }^{1}$ The remaining bins $i=2, \ldots, P Q$ are in no particular order yet.

For each bin $i$, an associated statistic $Z^{(i)}$ is generated as follows. First, the receiver performs the correlation of the received signal $r(t)$ with the $i$ th local replica

$$
x^{(i)}(t) \triangleq e^{\mathrm{j} 2 \pi \nu^{(i)} t} \sum_{n=-\infty}^{\infty} s_{1}\left(t-n T_{0}-\tau^{(i)}\right),
$$

The structure of $x^{(i)}(t)$ is the same as of $x_{1}(t)$, except from the modulating symbol sequence, which is assumed unknown to the receiver. Correlation during the coherent integration time $T$ leads to the correlator output

$$
Y^{(i)}[\ell]=\frac{1}{\sqrt{T}} \int_{\ell T}^{(\ell+1) T}\left(x^{(i)}(t)\right)^{*} r(t) \mathrm{d} t
$$

for coherent subintervals $\ell=0, \ldots, L-1$. We consider only integration times which are an integer multiple of the code

\footnotetext{
${ }^{1}$ Special cases, where the true code-phase and/or Doppler frequency lie exactly at a bin boundary, have probability measure zero.
}

period, i.e., $T=N T_{0}$. Secondly, the $L$ correlator outputs are noncoherently combined

$$
Z^{(i)}=\sum_{\ell=0}^{L-1}\left|Y^{(i)}[\ell]\right|^{2} .
$$

The total dwell time for the generation of $Z^{(i)}$ is $L T$. One possible receiver implementation is described in Fig. 1.

For the further analyses, it is useful to express the correlator output as the superposition of $K$ signal contributions and noise

$$
\begin{aligned}
Y^{(i)}[\ell] & =\sum_{k=1}^{K} X_{k}^{(i)}[\ell]+W^{(i)}[\ell] \\
X_{k}^{(i)}[\ell] & =\frac{1}{\sqrt{T}} \int_{\ell T}^{(\ell+1) T}\left(x^{(i)}(t)\right)^{*} x_{k}(t) \mathrm{d} t \\
W^{(i)}[\ell] & =\frac{1}{\sqrt{T}} \int_{\ell T}^{(\ell+1) T}\left(x^{(i)}(t)\right)^{*} w(t) \mathrm{d} t .
\end{aligned}
$$

Furthermore, we introduce the pulse's Fourier transform $H(\nu) \triangleq \int_{\mathbb{R}} h(t) e^{-\mathrm{j} 2 \pi \nu t} \mathrm{~d} t$ and its autocorrelation function $\varrho_{h}(t) \triangleq \int_{\mathbb{R}} h(\tau) h(\tau+t) \mathrm{d} \tau$, with normalization $\varrho_{h}(0)=T_{c}$.

\section{Decision criterion}

We consider only acquisition strategies based on threshold comparison, implemented either as serial or parallel search.

1) Serial search: Determine the starting index $j \in$ $\{1, \ldots, P Q\}$ (uniformly random or according to prior knowledge). Serially for $i=j, j-1, \ldots, 1, P Q, P Q-1, \ldots, j+1$, compare the statistic $Z^{(i)}$ to a fixed threshold $\lambda \geqslant 0$. As soon as $Z^{(i)}>\lambda$, immediately accept $H_{1}$, return the bin $i$, and terminate the search. If no statistic has exceeded the threshold after $P Q$ comparisons, accept $H_{0}$.

2) Parallel search: Compare all statistics to the threshold in parallel. Determine the subset $\mathcal{J}$ of all bins $j \in\{1, \ldots, P Q\}$ for which $Z^{(j)}>\lambda$. If $\mathcal{J}$ is non-empty, accept $H_{1}$ and return the $\operatorname{bin} i=\arg \max _{j \in \mathcal{J}} Z^{(j)}$ as code-phase/Doppler estimate. Otherwise, accept $H_{0}$.

\section{Probability of False Alarm}

Let $H_{0}$ be the true hypothesis. We say that a bin false alarm occurs in the $i$ th bin if $Z^{(i)}>\lambda$ for some $i \in\{1, \ldots, P Q\}$ (regardless of whether the actual search is terminated before reaching the $i$ th bin). Let the probability of this event be given by $P_{f}^{(i)}(\lambda)$. Moreover, a global false alarm is raised if at least one bin false alarm occurs (in that case, the receiver erroneously decides for $\left.H_{1}\right)$. We refer to $P_{f}^{(i)}(\lambda)$ as bin probability of false alarm (BPF), and to $P_{F}(\lambda)$ as global probability of false alarm (GPF).

In this section, we discuss various models for BPF/GPF, including state-of-the-art models and the novel model based on randomized SSCs. All of them are based on the notion of an effective noise floor, which means that they treat MAI as noiselike. The defining feature of each approach is the model for the effective noise floor $\mathcal{N}_{0}^{(i)}$ for each bin $i=1, \ldots, P Q$. 
Once $\mathcal{N}_{0}^{(i)}$ is specified, the $i$ th statistic is assumed to follow the cumulative distribution function (CDF)

$$
F_{Z}\left(z ; \mathcal{N}_{0}^{(i)}\right) \triangleq 1-e^{-\frac{z}{\mathcal{N}_{0}^{(i)}}} \sum_{\ell=0}^{L-1} \frac{1}{\ell !}\left(\frac{z}{\mathcal{N}_{0}^{(i)}}\right)^{\ell}, z \geqslant 0,
$$

independent for $i=1, \ldots, P Q$. This is a scaled version of the CDF of a central $\chi^{2}$-distribution with $2 L$ degrees of freedom. Given the effective noise floor for each bin, BPF and GPF are given by

$$
\begin{aligned}
P_{f}^{(i)}(\lambda) & \approx 1-F_{Z}\left(\lambda ; \mathcal{N}_{0}^{(i)}\right), \quad i=1, \ldots, P Q \\
P_{F}(\lambda) & \approx 1-\prod_{i=1}^{P Q} F_{Z}\left(\lambda ; \mathcal{N}_{0}^{(i)}\right) .
\end{aligned}
$$

Clearly, $P_{F}(\lambda)$ is the same for serial and parallel search [25].

\section{A. Neglecting Interference}

The simplest approximation is to ignore MAI and consider only the contribution of noise, i.e., to choose

$$
\mathcal{N}_{0}^{(i)}=N_{0}, \quad i=1, \ldots, P Q .
$$

This model is in fact accurate for a pure AWGN channel and a Nyquist pulse, whose autocorrelation function satisfies $\rho_{h}\left(n T_{c}\right)=0$ for all $n= \pm 1, \pm 2, \ldots$ In that case, it is a simple exercise to show that $W^{(i)}[\ell]$ are i.i.d. complex Gaussian with mean zero and variance $N_{0}$ for $\ell=0, \ldots, L-1$ [26]. For non-Nyquist pulses, the noise samples are actually correlated, but white noise is a common approximation. Notably, the assumption of independent statistics is strictly true if the bins have spacing $T_{c}$ in the code-phase domain and $1 / T$ in the Doppler domain, and an approximation otherwise [25].

The resulting approximation for the BPF can be expressed in terms of the generic CDF (13) as

$$
P_{f}^{(i)}(\lambda) \approx 1-F_{Z}\left(\lambda ; N_{0}\right)
$$

Importantly, this approximation does not depend on the bin index, so that (15) reduces to a simple exponentiation form $P_{F}(\lambda) \approx 1-\left(F_{Z}\left(\lambda ; N_{0}\right)\right)^{P Q}$.

\section{B. Standard SSC}

A common approximation is to calculate the MAI's noiseequivalent PSD $I_{0}$ in units of $\mathrm{W} / \mathrm{Hz}$, and then simply assume an increased effective noise floor for all bins

$$
\mathcal{N}_{0}^{(i)}=N_{0}+I_{0}, \quad i=1, \ldots, P Q .
$$

The convenience of this approach is that results for the pure AWGN channel can be reused, simply replacing $N_{0}$ by $N_{0}+$ $I_{0}$, to obtain the BPF

$$
P_{f}^{(i)}(\lambda) \approx 1-F_{Z}\left(\lambda ; N_{0}+I_{0}\right) .
$$

Again, this model does not depend on the bin index $i$. The MAI's noise-equivalent PSD is given by

$$
I_{0} \triangleq \operatorname{Var}\left[\sum_{k=2}^{K} X_{k}^{(i)}[\ell]\right]=\operatorname{SSC} \sum_{k=2}^{K} E_{k},
$$

Table I

SPECTRAL SEPARATION COEFFICIENT FOR COMMON PULSE SHAPES

\begin{tabular}{c|ccc}
$h(t)$ & REC & BOC & RRC \\
\hline $\mathrm{SSC}$ & $2 / 3$ & $1 / 3$ & 1
\end{tabular}

where $E_{k}=P_{k} T_{c}$. The ("self"-)SSC does not depend on the bin index $i$, but is determined solely by the pulse as

$$
\operatorname{SSC}=\frac{1}{T_{c}^{3}} \int_{-\infty}^{\infty}|H(\nu)|^{4} \mathrm{~d} \nu .
$$

The SSCs of some pulse shapes are stated in Table I, and the derivation of (20) can be found, for instance, in [?]. In the context of communications, this model has also been referred to as standard Gaussian approximation [26].

\section{Deterministic refined $S S C$ (SSC-D)}

The SSC-D models MAI with a much higher level of detail than the standard SSC. While slight variations can be observed among the proposed versions [20]-[22] in how they treat the dependency on code-phase, they are all essentially the same with regard to the (much more significant) Doppler dependency.

The initial approach is to calculate the MAI's noiseequivalent PSD conditioned on the constellation parameters of the interferers, $\boldsymbol{\nu} \triangleq\left[\nu_{2}, \ldots, \nu_{K}\right]^{T}$ and $\boldsymbol{\tau} \triangleq\left[\tau_{2}, \ldots, \tau_{K}\right]^{T}$. This leads to the conditional variance

$$
\mathcal{I}_{0}^{(i)} \triangleq \operatorname{Var}\left[\sum_{k=2}^{K} X_{k}^{(i)}[\ell] \mid \boldsymbol{\tau}, \boldsymbol{\nu}\right]
$$

and an effective noise floor

$$
\mathcal{N}_{0}^{(i)}=N_{0}+\mathcal{I}_{0}^{(i)}, \quad i=1, \ldots, P Q .
$$

Note that this effective noise floor varies from bin to bin. Therefore, the following equations (24)-(27), which are results from [22], [23] (and in similar form from [20], [21], [27]), need to be evaluated $P Q$ times per interferer. The conditional variance of the superposition of $K-1$ independent signals is the sum of condtional variances $\mathcal{I}_{0}^{(i)}=\sum_{k=2}^{K} \mathcal{I}_{k}^{(i)}$. For an interferer $k \neq 1$ affecting the $i$ th bin, a weighted SSC-D is obtained

$$
\begin{aligned}
\mathcal{I}_{k}^{(i)} & \triangleq \operatorname{Var}\left[X_{k}^{(i)}[\ell] \mid \tau_{k}, \nu_{k}\right] \\
& =\operatorname{SSC}\left(\tau^{(i)}-\tau_{k}, \nu^{(i)}-\nu_{k}\right) E_{k} .
\end{aligned}
$$

The SSC-D depends on the difference of the $i$ th codephase/Doppler candidate with respect to the $k$ th interferer's code-phase/Doppler and factorizes as

$$
\operatorname{SSC}(\tau, \nu)=\alpha_{I}(\tau) \beta_{I}(\nu)
$$

with a pulse interference function [22], [23], [27] and a code interference function [20]-[23], respectively,

$$
\begin{aligned}
& \alpha_{I}(\tau)=\sum_{m=-\infty}^{\infty} \frac{\rho_{h}^{2}\left(\tau-m T_{c}\right)}{T_{c}} \\
& \beta_{I}(\nu)=\sum_{n=1-N}^{N-1}\left(1-\frac{|n|}{M}\right)\left(1-\frac{|n|}{N}\right) \cos \left(2 \pi \nu n T_{0}\right),
\end{aligned}
$$




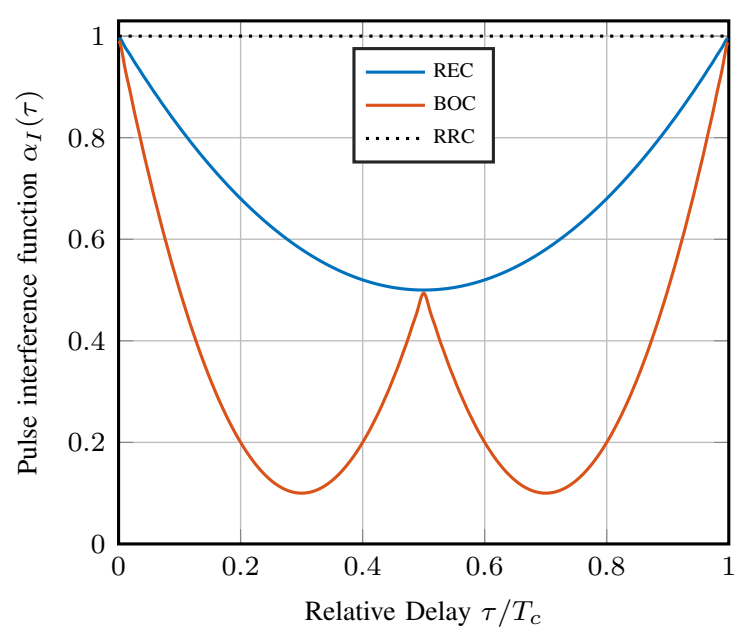

Fig. 2. Pulse interference function vs. relative delay for various pulse shapes: rectangular (REC), binary offset carrier (BOC), root-raised cosine (RRC).

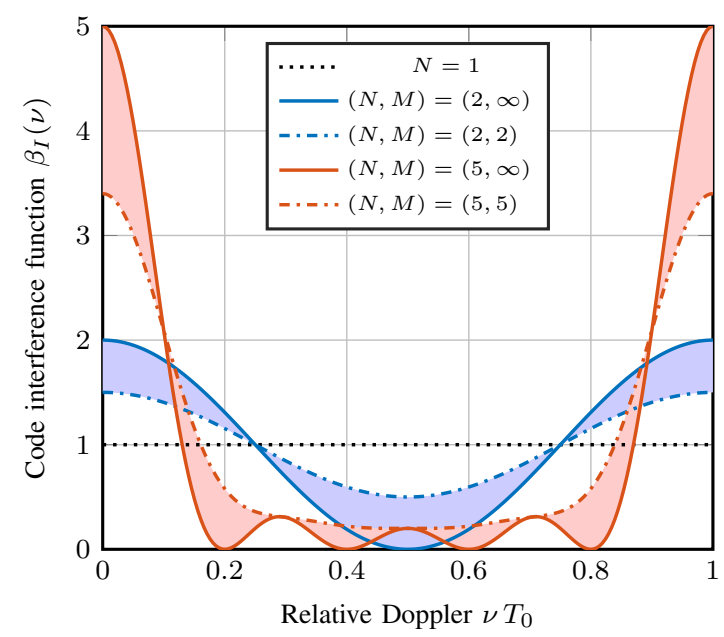

Fig. 3. Code interference function vs. relative Doppler. $N$ and $M$ are the number of code repetitions per coherent correlation and per symbol, respectively. Shaded areas indicate the cases where $N<M<\infty$.

where $N \leq M$. These interference functions are shown in Figs. 2, 3 .

Example. Consider Fig. 4. A static receiver observes a Walker(24/3/1) constellation [28]. Each in-view satellite transmits a pure pilot signal with PRN code length $N_{c}=341$, RRC pulse with zero roll-off, and chip duration $T_{c}=1 / 1.023 \mu \mathrm{s}$. The noise floor $N_{0}$ is $-204 \mathrm{dBW} / \mathrm{Hz}$, and the received powers $P_{k}$ are between $-160 \mathrm{dBW}$ and $-152 \mathrm{dBW}$, using the elevation dependent model from [29]. The SOI is transmitted from satellite $k=1$, whose pass takes about five hours and whose Doppler frequency $\nu_{1}$ is indicated by white dots. The coherent integration time is $20 \mathrm{~ms}$, hence we have $N=60$ code periods per coherent correlation and $M \rightarrow \infty$ (pure pilot). In Fig. 4, the effective noise floor $N_{0}+\mathcal{I}_{0}^{(i)}$ is shown as a function of a bin's Doppler candidate $\nu^{(i)}$ and time. The maximum contribution of interferer $k$ to the effective noise floor is equal to $N E_{k} \approx-194 \mathrm{dBW} / \mathrm{Hz}$, and occurs whenever the relative Doppler $\nu^{(i)}-\nu_{k}$ is approximately a multiple of $1 / T_{0}=3 \mathrm{kHz}$. This can happen for up to three interferers at the same time for

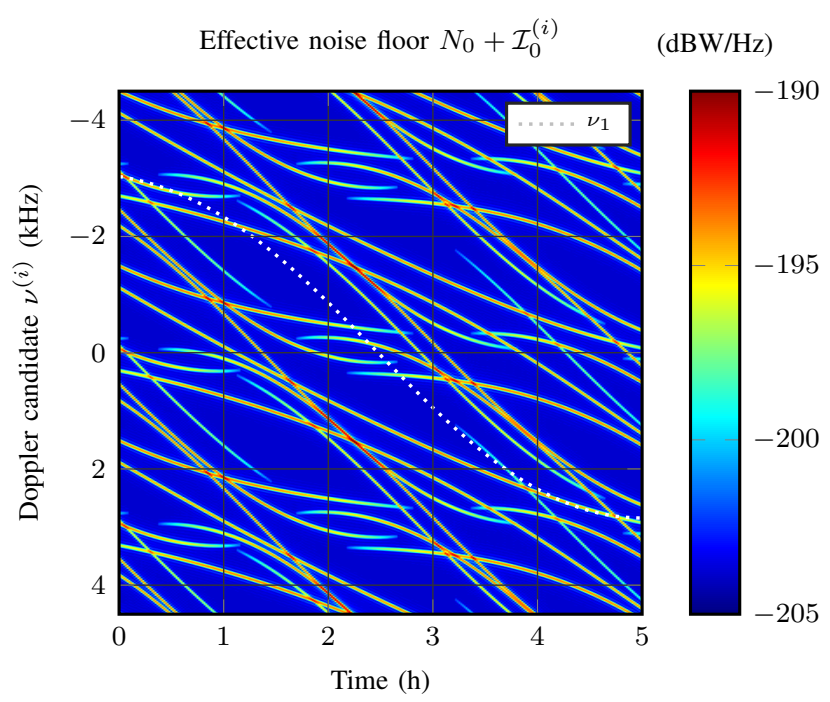

Fig. 4. Variation of the effective noise floor vs. Doppler bins during a satellite pass. The correct Doppler $\nu_{1}$ of the SOI is indicated by the dotted line.

the same Doppler candidate. As Doppler frequencies change slowly over time, such adverse conditions can last for several minutes.

The difficulty with the SSC-D model is the necessity to calculate not one, but up to $P Q$ effective noise floors. The BPF for bin $i$ using the SSC-D is

$$
P_{f}^{(i)}(\lambda) \approx 1-F_{Z}\left(\lambda ; N_{0}+\mathcal{I}_{0}^{(i)}\right), \quad i=1, \ldots, P Q .
$$

which depends on the bin index. Calculating all $P Q$ BPFs and the resulting GPF $P_{F}(\lambda)(15)$ is usually not practical for two reasons. Firstly, it is extremely cumbersome to compute $P Q$ effective noise floors, especially for a large number of bins. Second, $P_{F}(\lambda)$ and $P_{f}^{(i)}(\lambda)$ under the SSC-D are conditional probabilities, as they are treat the random interferer parameters as deterministic (conditional). As such, they may be very accurate for an instantaneous satellite constellation, but not representative of all possible cases. Nevertheless, this model provides useful insights and can be used to develop the following approximation superior to the standard SSC.

\section{Randomized refined SSC (SSC-R)}

Like in the previous section, we allow for a bin-dependent effective noise floor $\mathcal{N}_{0}^{(i)}=N_{0}+\mathcal{I}_{0}^{(i)}$. However, instead of using the exact conditional formula (22) for the MAI variances $\mathcal{I}_{0}^{(i)}, i=1, \ldots, P Q$, we assume they are i.i.d. random variables. Therefore, the CDF (13) becomes a compound probability distribution function. Suppose that

$$
\mathcal{I}_{0}^{(i)} \sim f_{\mathcal{I}_{0}}\left(\mathcal{I}_{0}\right), i=1, \ldots, P Q
$$

sampled from a single representative MAI distribution $f_{\mathcal{I}_{0}}\left(\mathcal{I}_{0}\right)$. A trivial distribution would be $f_{\mathcal{I}_{0}}\left(\mathcal{I}_{0}\right)=\delta\left(\mathcal{I}_{0}-I_{0}\right)$, which leads to the standard SSC model. Instead, we propose to construct a more representative distribution by the following two-step approach. 


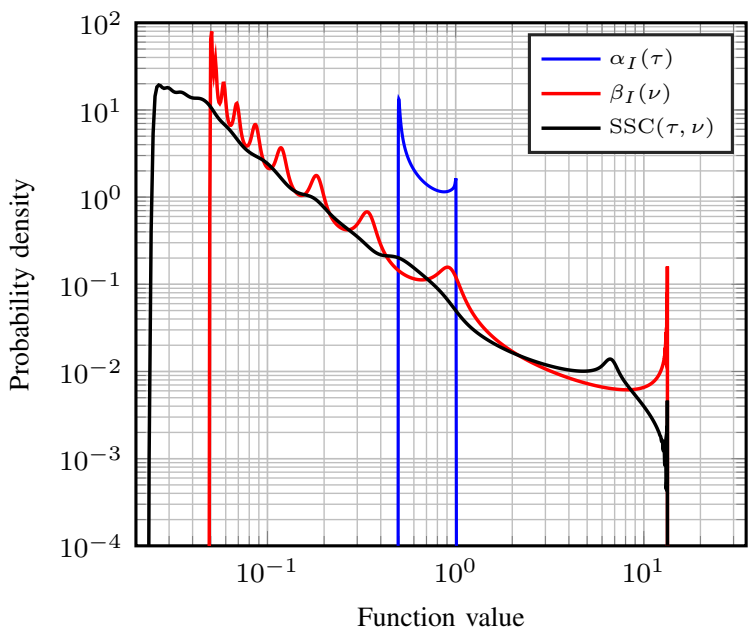

Fig. 5. PDF of the SSC-R and its two factors for an L1 C/A signal with coherent integration over $N=20$ code periods, assuming independent uniformly distributed code-phase and Doppler $(\tau, \nu)$.

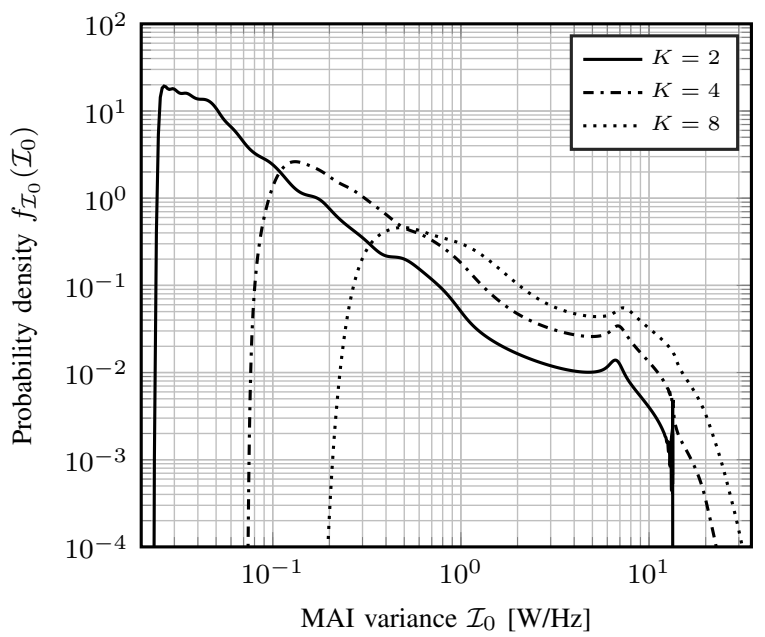

Fig. 6. PDF of MAI variance for $K-1$ interferers $\left(E_{2}=\ldots=E_{K}=1 \mathrm{Ws}\right)$ for an L1 C/A signal with coherent integration over $N=20$ code periods. The PDF for a single interferer corresponds to the PDF of the SSC in Fig. 5.

- Compute the PDF of the periodic function $\operatorname{SSC}(\tau, \nu)$ for uniform $\tau \sim \mathcal{U}\left(0, T_{c}\right)$ and $\nu \sim \mathcal{U}\left(0, T_{0}^{-1}\right)$. The result is the PDF of the SSC-R, which is shown in Fig. 5.

- Scale the obtained PDF by the energy $E_{k}$ of interferer $k$ to obtain the PDF $f_{\mathcal{I}_{k}}(\cdot)$ for $k=2, \ldots, K$. For the superposition of MAI from $K-1$ independent interferers, perform $K-2$ convolutions

$$
f_{\mathcal{I}_{0}}\left(\mathcal{I}_{0}\right)=\left(f_{\mathcal{I}_{2}} * \ldots * f_{\mathcal{I}_{K}}\right)\left(\mathcal{I}_{0}\right) .
$$

The result is shown in Fig. 6.

With $f_{\mathcal{I}_{0}}\left(\mathcal{I}_{0}\right)$, the conditioning in (28) can be removed to obtain the unconditional BPF

$$
P_{f}^{(i)}(\lambda) \approx 1-\int_{0}^{\infty} F_{Z}\left(\lambda ; N_{0}+\mathcal{I}_{0}\right) f_{\mathcal{I}_{0}}\left(\mathcal{I}_{0}\right) \mathrm{d} \mathcal{I}_{0} .
$$

This approximation does not depend on the bin index and only needs to be computed once per threshold $\lambda$. Note that this unconditional BPF is not representative for any particular bin, but for the ensemble of bins.

The assumption of i.i.d. MAI variances appears to be a strong one, and indeed does not account for the deterministic pattern that we observed in the example shown in Fig. 4. However, it is considerably more general than the standard SSC model, as it still accounts for the fact that the effective noise floor need not be the same across all bins. The PDF $f_{\mathcal{I}_{0}}\left(\mathcal{I}_{0}\right)$ is considered representative of MAI under all possible constellations, but could be refined for specific scenarios.

\section{Probability of Detection}

Let $H_{1}$ be the true hypothesis, i.e., the SOI is present. We say that global detection occurs if the receiver decides for $H_{1}$ and returns the correct bin $i=1$. The probability of this event is called global probability of detection (GPD). It is denoted by $P_{D}^{\downarrow}(\lambda)$ for serial search and by $P_{D}^{\|}(\lambda)$ for parallel search, respectively.

\section{A. Conditional GPD}

We define the $i$ th noncentrality energy as the energy that is delivered to the statistic $Z^{(i)}$ in the absence of MAI and noise

$$
\mathcal{E}^{(i)} \triangleq \sum_{\ell=0}^{L-1}\left|X_{1}^{(i)}[\ell]\right|^{2}, \quad i=1, \ldots, P Q .
$$

Ideally, $\mathcal{E}^{(1)}=P_{1} L T$ and $\mathcal{E}^{(2)}=\ldots=\mathcal{E}^{(P Q)}=0$. In practice, this is not the case: energy is lost in the correct bin and can leak into other bins due to various effects. In fact, the noncentrality energies are functions of $\tau_{1}, \nu_{1}$ and the states of the symbol sequence $\boldsymbol{b}_{1} \triangleq\left[b_{1}[0], \ldots, b_{1}[L N]\right]^{T}$ during the observation time. Therefore, $\mathcal{E}^{(1)}, \ldots, \mathcal{E}^{(P Q)}$ are statistically dependent random variables. Exemplarily, we determined the marginal distribution of the noncentrality energy $\mathcal{E}^{(1)}$ numerically in Fig. 7. It can be shown that [6]

$$
\mathcal{E}^{(i)}=\alpha_{C}\left(\tau^{(i)}-\tau_{1}\right), \beta_{C}\left(\nu^{(i)}-\nu_{1} ; \vartheta_{1} T_{0}+\tau_{1}, \boldsymbol{b}_{1}\right) P_{1} L T,
$$

with a code-phase correlation function

$$
\alpha_{C}(\tau)=\sum_{i=-\infty}^{\infty} \frac{\rho_{h}^{2}\left(\tau-i T_{0}\right)}{T_{c}^{2}}
$$

and a Doppler correlation function

$$
\beta_{C}(\nu ; \theta, \boldsymbol{b})=\frac{T_{c}^{2}}{T^{2}} \frac{\sin ^{2}(\pi \nu T)}{\sin ^{2}\left(\pi \nu T_{c}\right)}\left(1-\frac{2 X(\boldsymbol{b})}{L} \phi(\nu, \theta)\right),
$$

with the number of symbol transitions $X(\boldsymbol{b}) \in\{0, \ldots, L\}$ and an auxiliary function ${ }^{2}[6]$

$$
\phi(\nu, \theta)=\cot ^{2}(\pi \nu T)\left(\tan (\pi \nu T) \sin (2 \pi \nu \theta)-2 \sin ^{2}(\pi \nu \theta)\right) .
$$

If a pure pilot signal is considered, we can simply use $\beta_{C}(\nu) \triangleq$ $\left(T_{c} / T\right)^{2}\left(\sin (\pi \nu T) / \sin \left(\pi \nu T_{c}\right)\right)^{2}$. The energy loss and leakage effects are illustrated in Figs. 8, 9.

${ }^{2}$ Note that the auxiliary function becomes more complicated if $L>1$ and $N<M$, a case which is ommitted at this point for brevity but is treated in the thorough work of O'Driscoll [6]. 


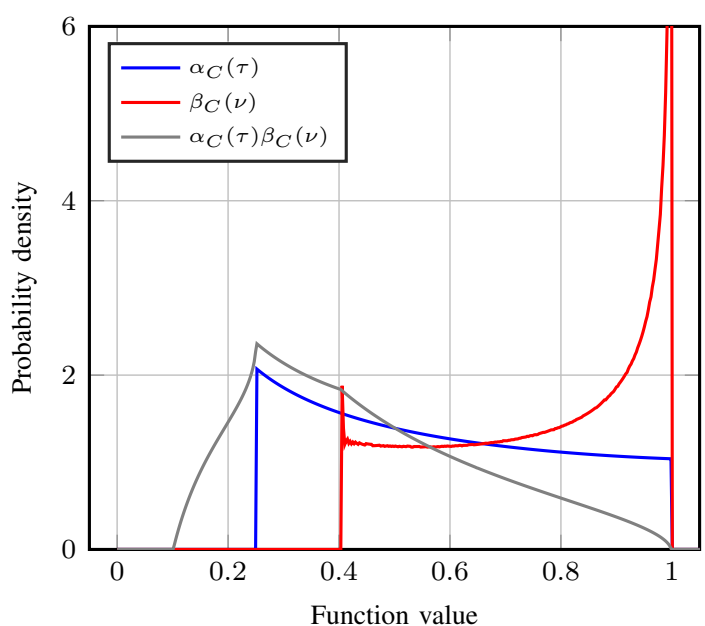

Fig. 7. PDF of the energy loss in the correct bin with code-phase spacing $\Delta \tau=T_{c}$ and Doppler spacing $\Delta \nu=1 / T$, for a BPSK pulse and pure pilot signaling $(b=1)$.

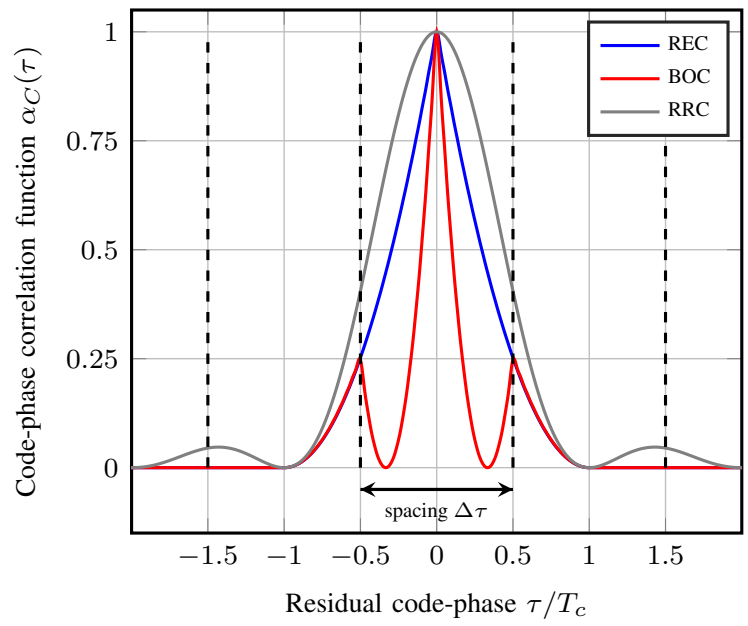

Fig. 8. Correlation vs. code-phase for various pulse shapes: rectangular (REC), binary offset carrier (BOC), root-raised cosine (RRC) with zero rolloff.

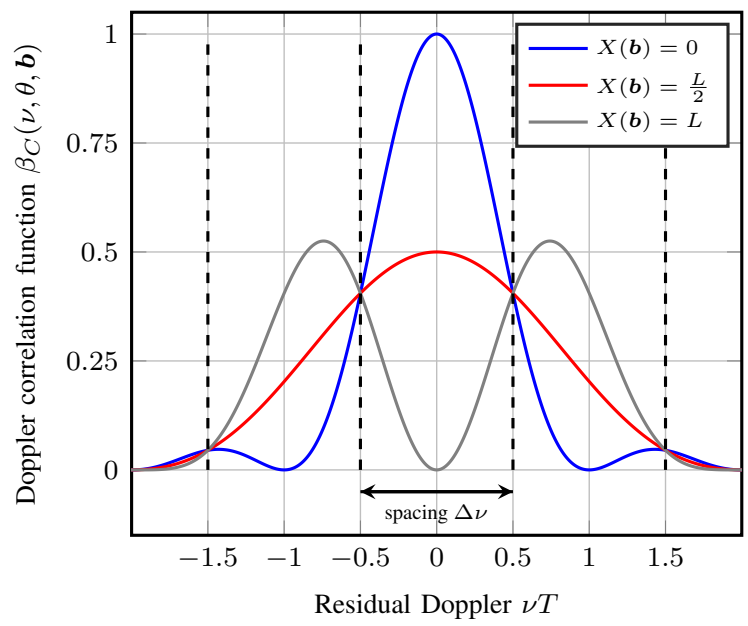

Fig. 9. Correlation vs. Doppler, with symbol boundaries in the middle of $L$ coherent subintervals $(\theta=T / 2)$. The number of symbol transitions is $X(\boldsymbol{b}) \in\{0,1, \ldots, L\}$.
Unlike MAI and noise, the contribution of the SOI to the statistic $Z^{(i)}$ is not Gaussian distributed. As proposed by [6], [30], we model this contribution by modifying the central $\chi^{2}$ distribution from (13) to a noncentral $\chi^{2}$-distribution with random noncentrality parameter (hence, a compound probability distribution). MAI and noise are the same as under $H_{0}$ and can be modeled by an effective noise floor $\mathcal{N}_{0}^{(i)}$, using one of the previously discussed interference models (Sections III-AIII-D). Adding the noncentral component to (13) leads to the generic $\mathrm{CDF}$ of $Z^{(i)}$ under $H_{1}$, given $\mathcal{E}^{(i)}$ and $\mathcal{N}_{0}^{(i)}$,

$F_{Z}^{\prime}\left(z ; \mathcal{E}^{(i)}, \mathcal{N}_{0}^{(i)}\right) \triangleq 1-Q_{L}\left(\sqrt{2 \frac{\mathcal{E}^{(i)}}{\mathcal{N}_{0}^{(i)}}}, \sqrt{2 \frac{z}{\mathcal{N}_{0}^{(i)}}}\right), z \geqslant 0$.

Here, $Q_{L}(\cdot, \cdot)$ denotes the $L$ th order Marcum Q-function [31]. This CDF is a scaled version of the CDF of a noncentral $\chi^{2}$ distribution with $2 L$ degrees of freedom and noncentrality parameter $2 \mathcal{E}^{(i)} / \mathcal{N}_{0}^{(i)}$. The according conditional bin probability of detection (BPD) is

$$
P_{d}^{(i)}(\lambda) \approx 1-F_{Z}^{\prime}\left(\lambda ; \mathcal{E}^{(i)}, \mathcal{N}_{0}^{(i)}\right)
$$

This probability is a compound probability in $\mathcal{E}^{(i)}$ and, if the randomized SSC is used, in $\mathcal{N}_{0}^{(i)}$.

Next, we compute the conditional GPD, given the vector of all noncentrality energies $\mathcal{E} \triangleq\left[\mathcal{E}^{(1)}, \ldots, \mathcal{E}^{(P Q)}\right]^{T}$ and the vector of effective noise floors $\mathcal{N}_{0} \triangleq\left[\mathcal{N}_{0}^{(1)}, \ldots, \mathcal{N}_{0}^{(P Q)}\right]^{T}$. Standard formulas [25] lead to the following results for serial or parallel search

$$
\begin{aligned}
& P_{D}^{\downarrow} \mid \mathcal{E}, \mathcal{N}_{0}=\frac{1-F_{Z}^{\prime}\left(\lambda ; \mathcal{E}^{(1)}, \mathcal{N}_{0}^{(1)}\right)}{P Q} \sum_{j=1}^{P Q} \prod_{i=2}^{j} F_{Z}^{\prime}\left(\lambda ; \mathcal{E}^{(i)}, \mathcal{N}_{0}^{(i)}\right) \\
& P_{D}^{\|} \mid \mathcal{E}, \mathcal{N}_{0}=\int_{\lambda}^{\infty} f_{Z}^{\prime}\left(z ; \mathcal{E}^{(1)}, \mathcal{N}_{0}^{(1)}\right) \prod_{i=2}^{P Q} F_{Z}^{\prime}\left(z ; \mathcal{E}^{(i)}, \mathcal{N}_{0}^{(i)}\right) \mathrm{d} z
\end{aligned}
$$

as long as we condition on $\mathcal{E}$ and $\mathcal{N}_{0}$. It remains to remove the conditioning on these parameters.

To simplify computation of the above equations, we assume $\mathcal{E}^{(i)} \approx 0$ if $\left|\tau^{(i)}-\tau_{1}\right|>T_{c}$ and $\left|\nu^{(i)}-\nu_{1}\right|>3 /(2 T)$. Therefore, apart from the the correct bin and some few adjacent bins, most bins are treated as central $\chi^{2}$-distributed.

\section{B. Removing the conditioning}

Starting from the conditional GPD (39) or (40), respectively, we remove the conditioning as follows.

1) Apply a MAI model to remove the conditioning on $\mathcal{N}_{0}$ :

- To neglect MAI (or use the standard SSC), simply set $\mathcal{N}_{0}^{(i)}=N_{0}\left(+I_{0}\right)$ for $i=1, \ldots, P Q$.

- To use the SSC-R, apply the law of total probability w.r.t. $\mathcal{N}_{0}$ : replace the conditional $\mathrm{CDF}$ $F_{Z}^{\prime}\left(z ; \mathcal{E}^{(i)}, \mathcal{N}_{0}^{(i)}\right)$ by the partly conditional CDF

$$
F_{Z}^{\prime}\left(z ; \mathcal{E}^{(i)}\right) \triangleq \int_{0}^{\infty} F_{Z}^{\prime}\left(z ; \mathcal{E}^{(i)}, N_{0}+\mathcal{I}_{0}\right) f_{\mathcal{I}_{0}}\left(\mathcal{I}_{0}\right) \mathrm{d} \mathcal{I}_{0}
$$


for all bins. For parallel search and $i=1$, replace the conditional PDF with $f_{Z}^{\prime}\left(z ; \mathcal{E}^{(1)}\right) \triangleq \frac{\mathrm{d}}{\mathrm{d} z} F_{Z}^{\prime}\left(z ; \mathcal{E}^{(1)}\right)$.

2) Remove the conditioning on $\mathcal{E}$ directly by averaging over the random SOI parameters $\tau_{1}, \nu_{1}$ and (if applicable) the possible symbol sequences $\boldsymbol{b}_{1},{ }^{3}$ using (33) as in [6].

\section{Numerical Results}

Unless stated otherwise, the following baseline setup is used for the remainder of this work.

- REC pulse, chip rate $1 / T_{c}=1.023 \mathrm{MHz}$;

- symbol rate $1 / T_{b}=0$ (pure pilot);

- code length $N_{c}=341 \Rightarrow$ code period $T_{0}=0.33 \mathrm{~ms}$;

- coh. integration time $T=1 \mathrm{~ms} \Rightarrow N=3$ code periods;

- number of noncoherent summations $L=1$;

- Doppler span $F_{0}=8 \mathrm{kHz}$;

- number of satellites $K=8$;

- noise floor $N_{0}=-204 \mathrm{dBW} / \mathrm{Hz}$;

- code-phase spacing $\Delta \tau=T_{c}$;

- Doppler spacing $\Delta \nu=1 / T$.

This setup leads to $P Q=341 \times 8=2728$ bins. Furthermore, we consider three characteristic scenarios, which are represented by three different power profiles $\left(P_{1}, \ldots, P_{K}\right)$ :

1) Balanced scenario: maximum received power [24] for all satellites: $P_{k}=-153 \mathrm{dBW}$ for $k=1, \ldots, K$.

2) Near-far scenario: maximum received power for $k=$ $2, \ldots, K$, minimum received power [24] for the SOI: $P_{1}=-158.5 \mathrm{dBW}$.

3) Weak signals scenario: very low received power for all signals: $P_{k}=-180 \mathrm{dBW}$ for $k=1, \ldots, K$.

As per definition, $P_{1}=0$ always under $H_{0}$. Note that the power profiles of Scenarios 1 and 2 are within system specifications [24], while only few systems are committed to service in weak signal conditions such as Scenario 3 [32]. However, these conditions are of some interest for navigation in space [11] or indoors [33].

\section{A. Baseline Setup}

Fig. 10 shows the BPF that is obtained with the baseline setup in Scenarios 1 and 2. In fact, there is not one BPF but many, depending on the bin's effective noise floor. The simulation results (markers) were obtained by Monte-Carlo simulations, randomly selecting one of the $P Q$ bins and determining its BPF. The BPF obtained with the SSC-R is representative not for any particular bin, but for the ensemble of bins, and matches well with the simulation results. The standard SSC leads to a slight underestimation of the BPF. For the SSC-D, only the BPF of the bin with the highest and lowest effective noise floor are shown. It is interesting to note that the BPFs under SSC and SSC-D appear as straight lines in a semilogarithmic plot, as the BPF for $L=1$ is an exponential function (13) of the threshold. By contrast, the SSC-R leads to a compound (mixture) BPF and appears as a slightly curved line. For the BPD in bin $i=1$, all SSC models lead to essentially the same results.

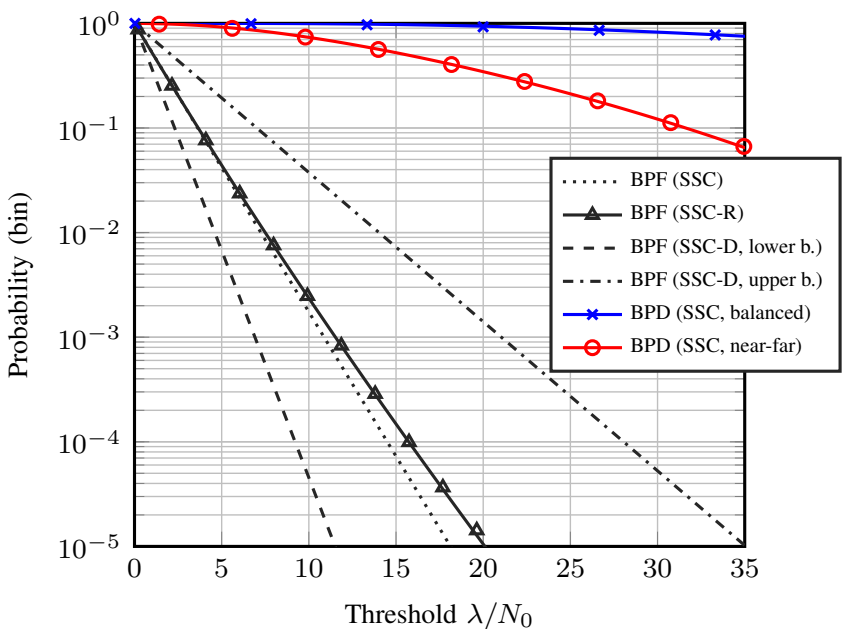

Fig. 10. Bin probabilities vs. threshold for Scenarios 1 (balanced) and 2 (near-far) with baseline setup $\left(N_{c}=341, T=1 \mathrm{~ms}\right)$. Markers represent simulation results.

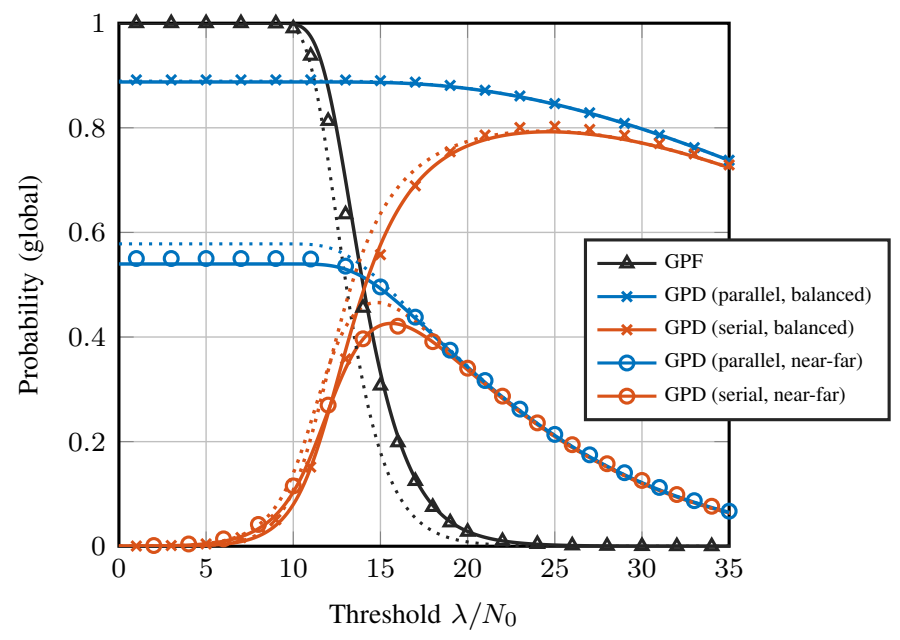

Fig. 11. Global probabilities vs. threshold for Scenarios 1 (balanced) and 2 (near-far) with baseline setup $\left(N_{c}=341, T=1 \mathrm{~ms}\right)$. Solid lines: SSC-R, dotted lines: SSC, markers: simulation results. $P Q=341 \times 8$ bins.

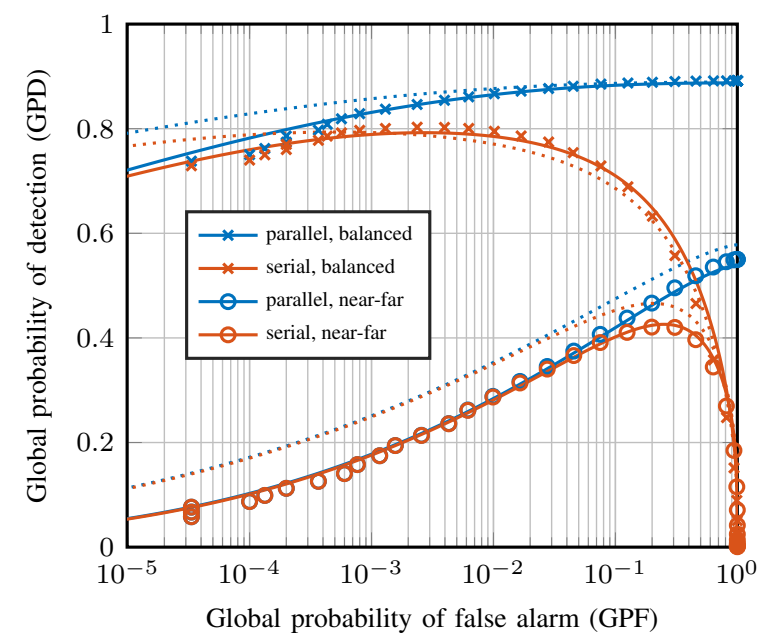

Fig. 12. ROC curve for Scenarios 1 (balanced) and 2 (near-far) with baseline setup $\left(N_{c}=341, T=1 \mathrm{~ms}\right)$. Solid lines: SSC-R, dotted lines: SSC, markers: simulation results. $P Q=341 \times 8$ bins. 


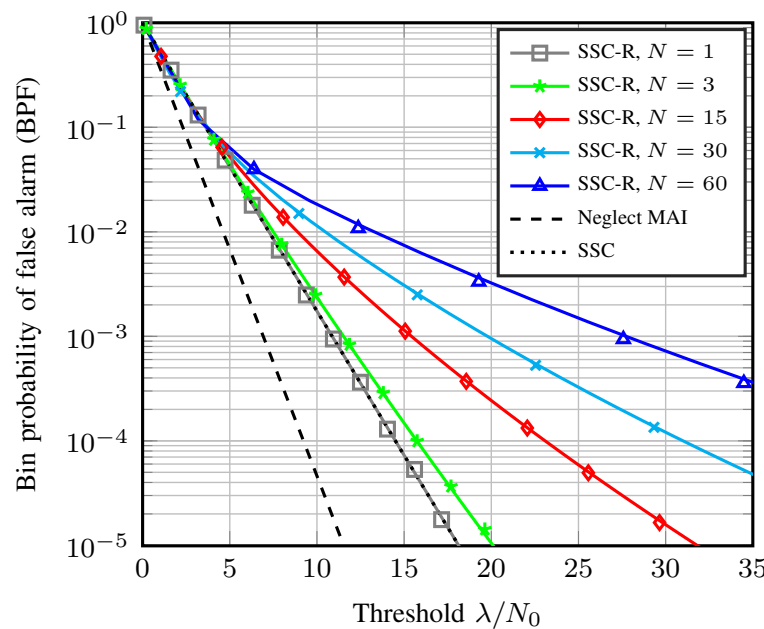

Fig. 13. BPF vs. threshold for Scenarios 1 (balanced) and 2 (near-far) with varying number $N$ of code periods per coherent integration. Markers represent simulation results.

Fig. 11 shows the global probabilities $P_{F}(\lambda), P_{D}^{\|}(\lambda), P_{D}^{\downarrow}(\lambda)$ as a function of the threshold, for Scenarios 1 and 2 . As these scenarios differ only in terms of the SOI power, $P_{F}(\lambda)$ is the same in either case, while detection is less likely in the near-far scenario. It can be observed that the standard SSC underestimates the GPF and slightly overestimates the GPD for all thresholds, while the SSC-R is in line with results from Monte-Carlo simulations. Plotting the GPD against the GPF, with the threshold $0 \leqslant \lambda<\infty$ as the varying parameter, leads to the ROC curve in Fig. 12. The receiver can operate at any point on the ROC curve by choosing the threshold accordingly.

\section{$B$. Increasing the coherent integration time $T$}

Increasing the coherent integration time is a good receiver side solution to enhance the acquisition reliability, especially in a near-far scenario. This leads to a proportional increase of the number of Doppler bins $Q$, while the number of codephase bins $P$ remains constant. As the code length $N_{c}$ and code period $T_{0}$ remain fixed, the receiver performs coherent integration over multiple code periods $N$. Increasing $N$ reveals the great weakness of the standard SSC: it depends only on $T$ but not on $N=T / T_{0}$. Thus, for large values of $N$, the BPF is grossly underestimated by the standard SSC, but correctly modeled by the SSC-R, as is shown in Fig. 13. The standard SSC also leads to a very overoptimistic ROC for $T=5 \mathrm{~ms}$ and $N=15$ in Fig. 14 .

\section{Increasing the number of noncoherent summations $L$}

For weak signals such as in Scenario 3, a good option to enhance reliability further is to increase the number of noncoherent summations $L$. Increasing the coherent integration time $T$ further and further would lead to an excessive number of Doppler bins, and is also difficult due to limitations of the

\footnotetext{
${ }^{3}$ There are $(L+1) M$ possible symbol sequences, as $\boldsymbol{b}$ can be parameterized in terms of the number of symbol transitions $X(\boldsymbol{b}) \in\{0, \ldots, L\}$ and the uniformly random initial symbol-phase $\vartheta_{1} \in\{1, \ldots, M\}$. If $L>1, X(\boldsymbol{b})$ follows a binomial distribution with $L$ trials and success probability $1 / 2$.
}

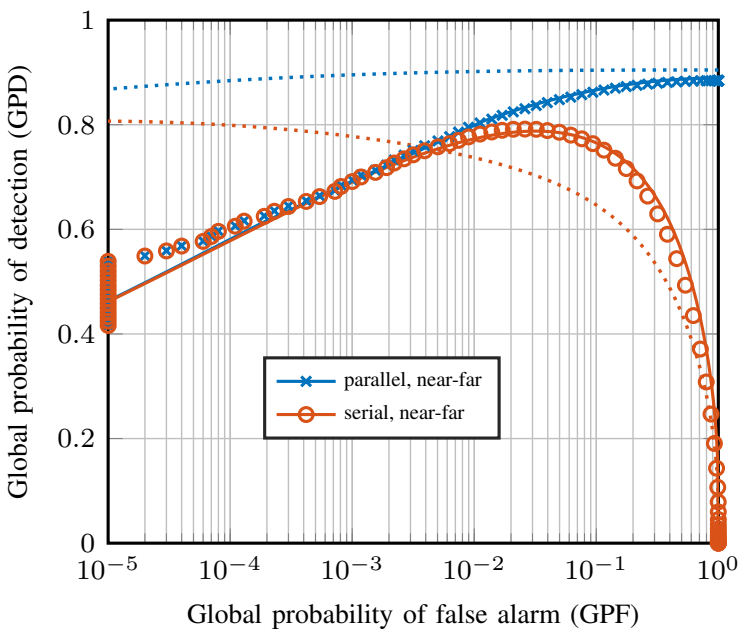

Fig. 14. ROC for Scenario 2 (near-far) with extended coherent integration time, $N_{c}=341, T=5 \mathrm{~ms}, N=15$. Solid lines: SSC-R, dotted lines: SSC, markers: simulation results. $P Q=341 \times 40$ bins.

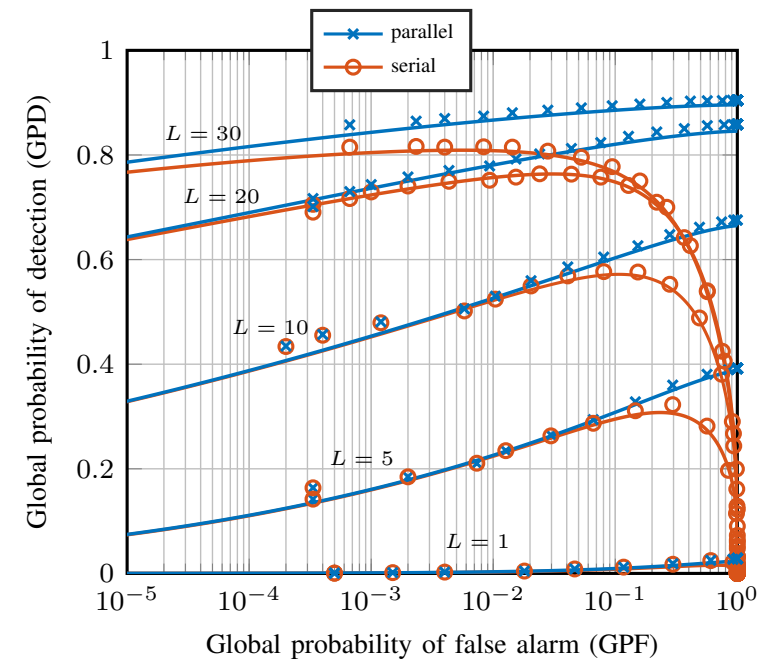

Fig. 15. ROC for Scenario 3 ("space") with $N=60$ coherent integrations and $L$ noncoherent summations. Solid lines: SSC, markers: simulation results. $P Q=341 \times 160$ bins.

receiver clock stability. Using more than $T=20 \mathrm{~ms}$ (hence $N=60$ in this case) is usually not practical. In Fig. 15, we show the ROC for $N=60$ and $L=1,5,10,20,30$. It can be observed that despite the large value of $N$, the standard SSC is already accurate (and virtually coincides with the SSC-R). This is due to the very low relative threshold $\lambda / N_{0}$ at the relevant operating points. As could be observed from Fig. 13, SSC and SSC-R lead to similar results as long as the ratio $\lambda / N_{0}$ is small.

\section{SignAl DESIGN: MINIMIZING PRN CODE LENGTH}

As an application example of the proposed methodology, we consider the design of a C/A signal for the European GNSS Galileo. This signal would complement the existing Galileo Open Service signals E1-B and E1-C transmitted at $1575.42 \mathrm{MHz}$ [24] and will be called E1-D in the following. E1-B and E1-C use a PRN code length of $N_{c}=$ 


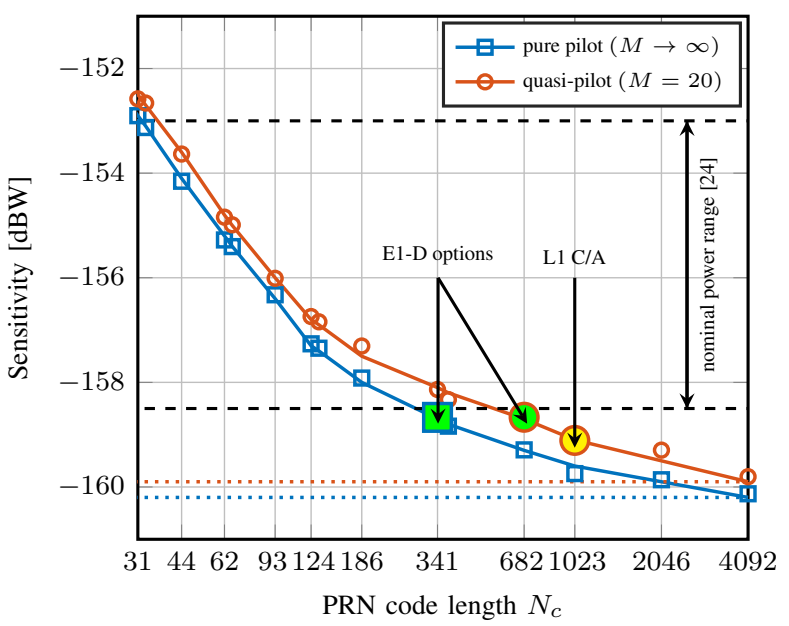

Fig. 16. Sensitivity of possible Galileo C/A signals vs. PRN code length, for a pure pilot and a quasi-pilot signal. The proposed signal designs and GPS L1 C/A are indicated. Solid lines: SSC-R, dotted lines: SSC, markers: simulation results.

4092, which is why we consider integer divisors $N_{c}=$ $2046,1023,682,372, \ldots$ as possible E1-D code lengths: this would allow for an easier handover from the C/A signal to the signals with longer PRN code. In terms of symbol rate, we consider the two options of a "pure pilot" signal and a "quasi-pilot" [13] signal with symbol duration $M T_{0}=20 \mathrm{~ms}$. The pulse shape is REC with chipping rate $1 / T_{c}=1.023$ MHz.

We aim to minimize the PRN code length over the set of integer divisors of 4092, while ensuring that the target reliability of $P_{D}^{\|}(\lambda)>80 \%, P_{F}(\lambda)<5 \%$ can be achieved for some $\lambda \geqslant 0$. The coherent integration time is $T=4 \mathrm{~ms}$, while $L=1$. Interferers $k=2, \ldots, 8$ are received with maximum power $P_{k}=-153 \mathrm{dBW}$. In Fig.16 (presented at [34]), we show the sensitivity, i.e., the necessary received power $P_{1}$ at which reliable acquisition is possible. For a PRN code length to be feasible, the sensitivity should not be above the nominal minimum received power level of $-158.5 \mathrm{dBW}$ [24]. As a result, we suggest the minimum code length of $N_{c}=341$ for a pure pilot signal or, alternatively, $N_{c}=682$ for a quasi-pilot signal with $50 \mathrm{~Hz}$ symbol rate. The slightly worse sensitivity of the quasi-pilot compared with a pure pilot is due to symbol transititons that occasionally lead to an energy loss. Note that the standard SSC is accurate only if $T=T_{0}$ and is therefore of no use for this application.

\section{CONCLUSION}

We argued in the introduction that C/A signals with short PRN code and low symbol rate are an attractive option to facilitate rapid, low energy acquisition, but are vulnerable to MAI. We demonstrated that some few Doppler search bins can be severely affected by C/A-on-C/A interference, and are thus more likely to lead to a global false alarm in the acquisition search. We proposed a methodology that can be used to assess the acquisition reliability in terms of the ROC curve (GPF plottes vs. GPD). The methodology is general and simple enough to be used in a signal design approach, where PRN code length, symbol rate, and other signal characteristics are flexible parameters to be selected. Applying the methodology to a signal design example, we showed that PRN code lengths on the order of 300-700 are feasible options for a Galileo C/A signal, which would reduce the number of search bins by a factor of $1.5-3$ as compared with L1 C/A. As an outlook, radio frequency compatibility of such a signal with co-existing (especially C/A-type) signals in the same frequency band should be assessed, using models to assess cyclostationary intersystem MAI [22].

\section{REFERENCES}

[1] E. D. Kaplan and C. Hegarty, Understanding GPS: principles and applications. Boston: Artech House, 2006.

[2] U. Cheng, W. Hurd, and J. Statman, "Spread-spectrum code acquisition in the presence of Doppler shift and data modulation," IEEE Transactions on Communications, vol. 38, no. 2, pp. 241-250, Feb. 1990.

[3] F. S. T. Van Diggelen, A-GPS: Assisted GPS, GNSS, and SBAS. Artech House, 2009.

[4] K. Chen, G. Tan, J. Cao, M. Lu, and X. Fan, "Modeling and Improving the Energy Performance of GPS Receivers for Location Services," IEEE Sensors Journal, vol. 20, no. 8, pp. 4512-4523, Apr. 2020.

[5] D. Borio, L. Camoriano, and L. Lo Presti, "Impact of GPS acquisition strategy on decision probabilities," IEEE Transactions on Aerospace and Electronic Systems, vol. 44, no. 3, pp. 996-1011, Jul. 2008.

[6] C. O'Driscoll, "Performance analysis of the parallel acquisition of weak GPS signals," Ph.D. dissertation, University College Cork, 2007.

[7] "Power-efficient positioning for the Internet of Things: merging GNSS with low power connectivity solutions: white paper," European GNSS Agency, Publications Office of the EU, Tech. Rep., 2020.

[8] G. Corazza, "On the MAX/TC criterion for code acquisition and its application to DS-SSMA systems," IEEE Transactions on Communications, vol. 44, no. 9, pp. 1173-1182, Sep. 1996.

[9] A. Coenen and D. van Nee, "Novel fast GPS/GLONASS codeacquisition technique using low update rate FFT," Electronics Letters, vol. 28, no. 9, p. 863, 1992.

[10] F. van Diggelen, "Redoing GNSS from Scratch - The Perfect System," St. Louis, Missouri, Sep. 2020, pp. 3199-3209.

[11] S. Wallner, "Galileo System and Signal Evolution," International Technical Symposium on Navigation and Timing, Toulouse, France, Nov. 2017.

[12] M. Paonni and M. Bavaro, "On the Design of a GNSS Acquisition Aiding Signal," in Proceedings of the 26th International Technical Meeting of the Satellite Division of The Institute of Navigation (ION GNSS+ 2013), Nashville, Tennessee, Sep. 2013, pp. 1445-1456.

[13] M. Paonni, M. Anghileri, D. Fontanella, and B. Eissfeller, "Quasi-Pilot Signals: Improving Sensitivity and TTFF without Compromises," in Proceedings of the 24th International Technical Meeting of the Satellite Division of The Institute of Navigation (ION GNSS 2011), Portland, Oregon, Sep. 2011, pp. 1254-1263.

[14] S. Wallner, J. A. Garcia Molina, G. L. Risueno, J. Hahn, F. Jean Jeacques, F. Soualle, T. Schmitt, G. da Broi, M. Ouedraogo, T. Woerz, G. de Pasquale, C. Vazquez, and M. Paonni, "Novel Concepts on GNSS Signal Design serving Emerging GNSS User Categories: Quasi-Pilot Signal," in European Navigation Conference (ENC) 2020. Dresden, Germany: DGON, Nov. 2020.

[15] T. A. Stansell, J. W. Betz, F. van Diggelen, and S. Kogure, "Proposed Evolution of the C/A Signal," in Proceedings of the 28th International Technical Meeting of the Satellite Division of The Institute of Navigation (ION GNSS+ 2015), Tampa, Florida, Sep. 2015, pp. 1807-1825.

[16] F. Van Diggelen, "Who's Your Daddy? Why GPS Will Continue to Dominate Consumer GNSS," Inside GNSS, vol. 9, no. 2, Mar. 2014.

[17] S. Qaisar and A. Dempster, "Cross-correlation performance assessment of global positioning system (GPS) L1 and L2 civil codes for signal acquisition," IET Radar, Sonar \& Navigation, vol. 5, no. 3, p. 195, 2011.

[18] J. W. Betz, "Effect of Narrowband Interference on GPS Code Tracking Accuracy," in Proceedings of the 2000 National Technical Meeting of The Institute of Navigation, Anaheim, CA, Jan. 2000, pp. 16-27.

[19] _ "Effect of Partial-Band Interference on Receiver Estimation of C/NO: Theory," in Proceedings of the 2001 National Technical Meeting of The Institute of Navigation, Long Beach, CA, Jan. 2001, pp. 817-828. 
[20] C. J. Hegarty, "A simple model for GPS C/A-code self-interference," NAVIGATION, vol. 67, no. 2, pp. 319-331, Jun. 2020

[21] C. O'Driscoll and J. Fortuny-Guasch, "On the Determination of C/A Code Self-Interference with Application to RFC Analysis and Pseudolite Systems," in Proceedings of the 25th International Technical Meeting of The Satellite Division of the Institute of Navigation (ION GNSS 2012), Nashville, TN, Sep. 2012, pp. 3620-3631.

[22] C. Enneking, F. Antreich, L. Krieger, and A. L. F. de Almeida, "Gaussian Approximations for Intra- and Intersystem Interference in RNSS," IEEE Communications Letters, vol. 23, no. 7, pp. 1198-1201, Jul. 2019, conference Name: IEEE Communications Letters.

[23] C. Enneking, F. Antreich, M. M. Appel, and A. L. de Almeida, "Pure Pilot Signals: How Short can we Choose GNSS Spreading Codes?" in 2019 International Technical Meeting of The Institute of Navigation, Reston, Virginia, Feb. 2019, pp. 925-935.

[24] European GNSS (Galileo) Open Service: Signal-in-Space Interface Control Document, European Union, OS SIS ICD Issue 1.3, Interface Specification, 2016.

[25] D. Borio, "A Statistical Theory for GNSS Signal Acquisition," Ph.D. dissertation, Politecnico di Torino, 2008.

[26] G. Zang and C. Ling, "Performance evaluation for band-limited DSCDMA systems based on simplified improved Gaussian approximation," IEEE Transactions on Communications, vol. 51, no. 7, pp. 1204-1213, Jul. 2003.

[27] J. H. Cho and J. S. Lehnert, "An optimal signal design for bandlimited asynchronous DS-CDMA communications," IEEE Transactions on Information Theory, vol. 48, no. 5, pp. 1172-1185, May 2002.

[28] European GNSS (Galileo) Initial Services: Open Service, European Union, OS SDD Issue 1.0, Service Definition Document, 2016.

[29] "Assessment of Radio Frequency Interference Relevant to the GNSS L1 Frequency Band," RTCA, Inc., DO-235, Mar. 2008.

[30] M. Foucras, O. Julien, C. Macabiau, B. Ekambi, and F. Bacard, "Probability of detection for gnss signals with sign transitions," IEEE Transactions on Aerospace and Electronic Systems, vol. 52, no. 3, pp. 1296-1308, 2016.

[31] J. Marcum, "A statistical theory of target detection by pulsed radar," IRE Transactions on Information Theory, vol. 6, no. 2, pp. 59-267, 1960.

[32] "NAVSTAR GPS Space Segment/Navigation User Interfaces," IS-GPS200, Rev. L, GPS Enterprise, El Segundo, CA, May 2020.

[33] N. Ziedan, GNSS receivers for weak signals. Boston, Lodon: Artech House, 2006.

[34] C. Enneking, F. Antreich, and A. L. F. de Almeida, "GNSS Acquisition Performance of Short Spreading Codes," in 33rd International Technical Meeting of the Satellite Division of The Institute of Navigation (ION GNSS+ 2020), Oct. 2020, pp. 1238-1260. 